\title{
GINA 2014: Licht und Schatten
}

\author{
GINA 2014: Yin and Yang
}

Autor

Institut
P. Kardos

Gemeinschaftspraxis \& Zentrum für Allergologie, Pneumologie \& Schlafmedizin eingereicht $\quad 1.10 .2014$ akzeptiert nach Revision 16.10.2014

\section{Bibliografie}

DOI http://dx.doi.org/

10.1055/s-0034-1390903

Pneumologie 2014; 68: 784-787

(c) Georg Thieme Verlag KG Stuttgart · New York ISSN 0934-8387

\section{Korrespondenzadresse} Dr. Peter Kardos

Gemeinschaftspraxis \& Zentrum für Allergologie

Pneumologie \& Schlafmedizin Scheffelstraße 33 60318 Frankfurt/Main kardos@lungenpraxis-maingau. de

\section{Zusammenfassung \\ $\nabla$}

Nach acht Jahren hat die Global Initiative for Asthma (GINA) ein umfassendes Update ihrer Empfehlungen vorgelegt. Im Mai 2014 wurde die neue Version online gestellt [www.ginasthma.org] und beim Kongress der European Respiratory Society im September dann offiziell präsentiert. Die Neuerungen beginnen bereits auf Seite 1: GINA ist keine Leitlinie, sondern eine "Global Strategy“. Dies wurde bei der Präsentation auf dem ERSKongress in München betont, was den in Richtung des pragmatischen Ansatzes veränderten Anspruch dokumentiert (und möglicherweise die Furcht vor Haftungsfragen).

Aus pneumologischer Sicht bringt GINA 2014 eine Reihe beachtenswerter, aber zum Teil auch diskussionswürdiger Veränderungen. Die wichtigste Änderung ist die erweiterte Zielsetzung für das Asthma-Management: Nicht mehr allein Symptomkontrolle („Asthmakontrolle“) soll angestrebt werden, sondern, analog zu den GOLD-Empfehlungen für die COPD, die Abschätzung von zukünftigen Risikofaktoren. Risikofaktoren können den Krankheitsverlauf verschlechtern, indem sie Exazerbationen begünstigen, zur Fixierung der Atemflusslimitierung beitragen oder das Nebenwirkungsrisiko erhöhen. Dazu zählen z.B. Rauchen und Adipositas, schlechte Inhalationstechnik, die Notwendigkeit häufiger Therapien mit oralen Kortikosteroiden (OCS) oder hohen Dosen inhalativer Kortikosteroide (ICS). Neu ist die ausdrückliche Empfehlung der Bestimmung der (absoluten) Eosinophilenzahl im Blut, eine sehr einfache und in Deutschland überall verfügbare, bislang aber für die Risikoabschätzung beim Asthma wenig praktizierte Untersuchung. Sie ist darüber hinaus zur Phänotypisierung des Asthmas dringend erforderlich, um bald verfügbare Biologika wie Anti-IL5 Antikörper sinnvoll im Rahmen der

\section{Abstract \\ $\nabla$}

After 8 years the Global Initiative for Asthma (GINA) presented a fully revised report. In May 2014 the new GINA was published online [www. ginasthma.org]. On a live GINA Session at the European Respiratory Society (ERS) conference 2014 in Munich members of the board of directors and of the science committee presented the new contents, e.g. the GINA statement from page one, that GINA is "Not a guideline, but a practical approach to managing asthma in clinical practice" - was explicitly emphasized on the ERS. This may reflect a changing claim towards a more pragmatic attempt (but probably also the fear of liability).

personalisierten Asthmabehandlung einsetzen zu können.

Sehr erfreulich ist, dass die unsäglichen Schweregrad-Definitionen früherer GINA-Versionen ( $\bullet$ Tab. 1) verlassen wurden, die eigentlich nur für das unbehandelte Asthma galten und nichtsdestotrotz in hochkarätigen Publikationen verwirrend falsch verwendet wurden. Noch die ATS/ ERS-Leitlinie „Severe Asthma“ [1] vom Februar 2014 widersprach der damals gültigen GINADefinition, als sie schweres Asthma wie folgt definierte: „Requires treatment with guidelines suggested medications for GINA steps 4-5 asthma (high dose ICS and LABA or leukotriene modifier/ theophylline) for the previous year or systemic CS for over $50 \%$ of the previous year to prevent it from becoming uncontrolled or which remains uncontrolled despite this therapy."

GINA 2014 fand eine der klinischen Praxis angepasste Definition der Schweregrade ( Tab.2). Das geht auch mit einer Änderung des therapeutischen Stufenschemas einher ( $\bullet$ Abb.1) - dazu später mehr. Künftig soll der Schweregrad erst nach einigen Monaten unter Controller-Therapie retrospektiv anhand des Medikationsbedarfs fest- 
Tab. 1 GINA 2004: Definition der Asthma-Schweregrade.

\section{Current Treatment Step}

\begin{tabular}{|c|c|c|c|}
\hline & \multicolumn{3}{|c|}{ Current Treatment Step } \\
\hline & Step 1: Intermittent & Step 2: Mild Persistent & Step 3: Moderate Persistent \\
\hline Patient Symptoms and Lung Function on Current Therapy & \multicolumn{3}{|c|}{ Level of Severity } \\
\hline $\begin{array}{l}\text { Step 1: Intermittent } \\
\text { Symptoms less than once a week } \\
\text { Brief exacerbations } \\
\text { Nocturnal symptoms not more than twice a month } \\
\text { Normal lung function between episodes }\end{array}$ & Intermittent & Mild Persistent & Moderate Persistent \\
\hline $\begin{array}{l}\text { Step 2: Mild Persistent } \\
\text { Symptoms more than once a week but less than once a day } \\
\text { Nocturnal symptoms more than twice a month but less than } \\
\text { once a week } \\
\text { Normal lung function between episodes }\end{array}$ & Mild Persistent & Moderate Persistent & Severe Persistent \\
\hline $\begin{array}{l}\text { Step 3: Moderate Persistent } \\
\text { Symptoms daily } \\
\text { Exacerbations may affect activity and sleep } \\
\text { Nocturnal symptoms at least once a week } \\
60 \%<\mathrm{FEV} V_{1}<80 \% \text { predicted OR } \\
60 \%<\mathrm{PEF}<80 \% \text { of personal best }\end{array}$ & Moderate Persistent & Severe Persistent & Severe Persistent \\
\hline $\begin{array}{l}\text { Step 4: Severe Persistent } \\
\text { Symptoms daily } \\
\text { Frequent exacerbations } \\
\text { Frequent nocturnal asthma symptoms } \\
\mathrm{FEV}_{1} \leq 60 \% \text { predicted OR } \\
\mathrm{PEF} \leq 60 \% \text { of personal best }\end{array}$ & Severe Persistent & Severe Persistent & Severe Persistent \\
\hline
\end{tabular}

$\mathrm{FEV}_{1}$ : forciertes expiratorisches Volumen in der 1. Sekunde; PEF: peak expiratory flow.

Tab. 2 GINA 2014 Abschätzung des Asthma-Schweregrades.

Wie wird der Schweregrad ermittelt?
- Retrospektiv anhand des Medikationslevels, das notwendig ist, um
Symptome und Exazerbationen zu kontrollieren
Kategorien
- Mildes Asthma: gut kontrolliert mit Stufe 1 oder 2 (SABA bei Bedarf
oder niedrig dosiertes ICS)
- Moderates Asthma: gut kontrolliert mit Stufe 3 (ICS/LABA niedrig
dosiert)
- Schweres Asthma: benötigt Stufe 4/5 (ICS/LABA in moderater bis
hoher Dosis \pm Add-on-Medikation) oder bleibt trotz dieser Therapie
unkontrolliert

SABA: short acting betaagonist; ICS: inhaled corticosteroid; LABA: long acting betaagonist; LTRA: Leukotrien-Rezeptor-Antagonist; OCS: oral corticosteroid.

gestellt werden: Mildes Asthma benötigt einen kurz wirksamen Beta2-Agonisten bei Bedarf bzw. ein niedrig dosiertes ICS, d.h. mildes Asthma kann auf Therapiestufe 1 oder 2 kontrolliert werden. Moderates Asthma wird mit ICS plus lang wirksamem Beta2-Agonisten (LABA) gut kontrolliert (Stufe 3), schweres Asthma benötigt moderate bis hohe ICS-Dosen plus LABA plus eventuell weitere Controller (Stufe 4 und 5).

Nach Herausnahme der Lungenfunktion aus den Kriterien, die die Asthmakontrolle bestimmen, verlassen wir uns nun auf rein subjektive Angaben des Patienten. Bei einer Erkrankung wie COPD, die eine persistente - zwar laut COPD-Definition behandelbare, aber wenn wir ehrlich sind, nur wenig veränderbare Einschränkung bedeutet und wo das Befinden des Patienten die Hauptrolle spielt, mag das richtig sein. Beim Asthma dagegen könnte der Verzicht auf objektive Lungenfunktionsdaten zur Überschätzung der Asthmakontrolle führen, entsprechende Daten über Unterschätzung der Schwere der Bronchialobstruktion durch den Patienten liegen in der Literatur vor [2,3].
Bemerkenswert ist die neue Asthma-Definition, die zwar zu Recht die Heterogenität der Asthmaerkrankung betont und Symptome und Atemflusslimitierung als diagnostische Kriterien aufführt, aber die bronchiale Hyperreagibilität (BHR) unter den Tisch fallen lässt. Selbst wenn im Text versteckt Hinweise auf die Nützlichkeit der Prüfung der bronchialen Hyperreagibilität gegeben wurden, ist für uns niedergelassene Fachärzte die Streichung dieses Kriteriums aus der Asthma-Definition ein herber Verlust. Denn bisher haben wir bei $80 \%$ unserer jüngeren Asthmapatienten, die mit weitgehend normaler Lungenfunktion in die Praxis kommen, das Asthma primär anhand von BHR und Symptomen diagnostiziert. Es ist verwunderlich, dass dieses wichtige Diagnosekriterium zu einem Zeitpunkt in den Hintergrund geraten ist, als erstmals in GINA auf die häufig inkorrekte Asthmadiagnose hingewiesen wird [4-7]: 25-35\% der in der Allgemeinpraxis mit Asthmamedikamenten behandelten Patienten haben gar kein Asthma.

Die neue Definition stellt uns zudem vor das Problem, dass sie die Abgrenzung gegen die COPD erschwert. Danach finden sich mehr Gemeinsamkeiten als Unterschiede zwischen den Krankheitsbildern: Beiden liegen chronische Entzündungsprozesse zugrunde, ohne dass näher differenziert wird, welcher Art diese Entzündung ist. Beide gehen mit Obstruktion einher. Als Differenzierungsmerkmale sind vor allem die Variabilität der Obstruktion sowie Symptommuster geblieben (die Reversibilität als Kriterium wurde aus der Definition verbannt), die jedoch ebenfalls keine „harten“ diagnostischen Kriterien darstellen, sondern lediglich die Wahrscheinlichkeit pro Asthma erhöhen oder erniedrigen. $\mathrm{Zu}$ diesen Unsicherheiten passt, dass GINA dem Asthma-COPDOverlap-Syndrom, kurz ACOS, ein eigenes Dokument gewidmet hat, das praktisch vollständig ohne Evidenz auskommt, gezwungenermaßen, denn es gibt keine qualitativ hochwertigen Studien zu dieser Mischform von Asthma und COPD. Im Gegenteil: Praktisch alle modernen Asthma- und COPD-Studien sorgten für den 


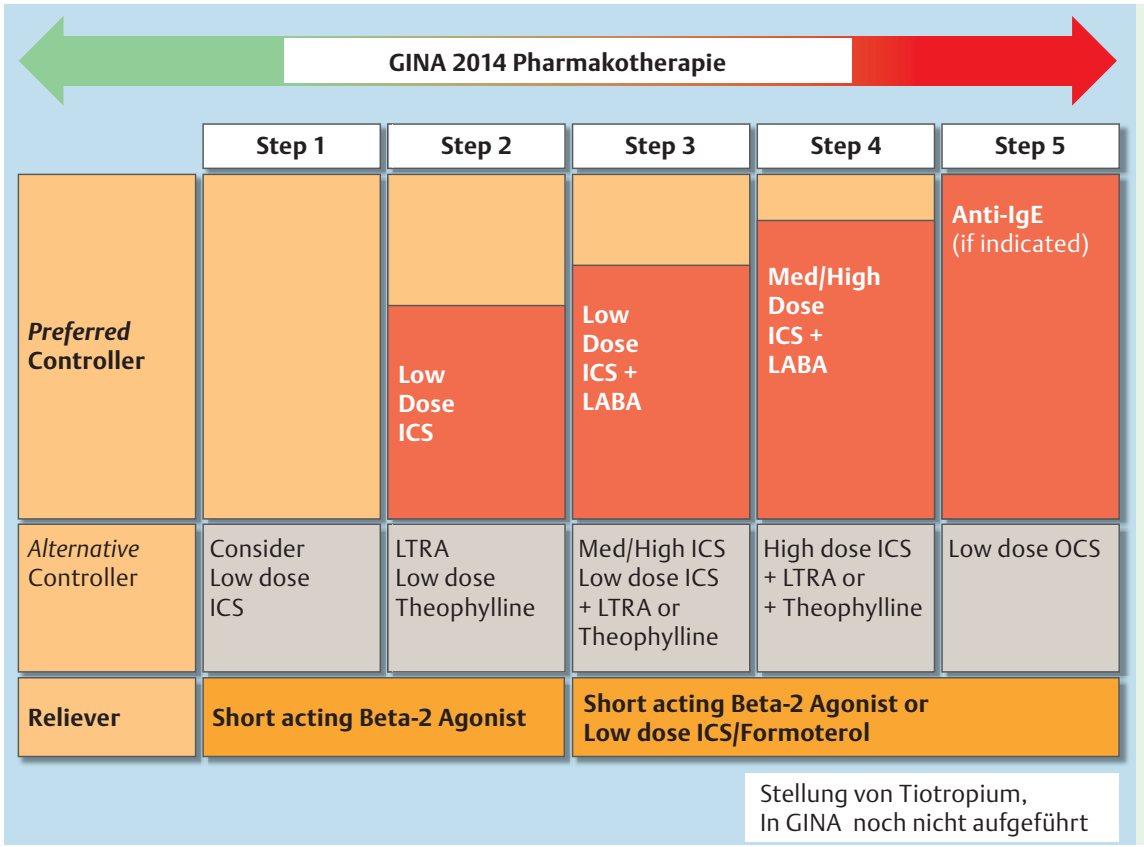

Abb. 1 GINA 2014 Pharmakotherapie.

sorgfältigen Ausschluss von Patienten, die gewisse Kriterien der anderen Erkrankung erfüllt haben. So wurden Raucher mit über 10 Packungsjahren aus allen Asthmastudien ausgeschlossen, ungeachtet der Tatsache, dass der Anteil der Raucher unter Asthmapatienten exakt dem Anteil der Raucher in der Allgemeinbevölkerung entspricht und daher solche Ausschlusskriterien die externe Validität dieser Studien stark verringert haben [8].

Die schwache Evidenzlage schlägt sich bereits in der äußerst vagen Definition von ACOS nieder, der zufolge ACOS „identifiziert wird durch die Charakteristika, die es mit Asthma und COPD teilt“. Zweifellos ist es richtig anzuerkennen, dass es Patienten gibt, die sowohl an Asthma als auch an COPD leiden. Inwieweit eine neue Entität zu einem Zeitpunkt geschaffen werden musste, zu dem für diese Patienten noch keine evidenzbasierten Kriterien für Diagnostik und Therapie existieren, kann diskutiert werden. Vorteilhaft ist jedoch, dass die wissenschaftliche Gemeinschaft gefordert wird, um Krankheitsbild, Diagnostik und Therapie besser zu charakterisieren. Wir als Pneumologen müssen aufpassen, dass nicht Patienten aus diagnostischer Unsicherheit in die ACOS-Schublade einsortiert werden.

Positiv zu vermerken ist, dass endlich auch ein komplettes Kapitel für das frühkindliche Asthma (unter 5 Jahren) verfügbar ist.

Der neue GINA-Report betont sehr stark die Wichtigkeit von Inhalatoren- und Inhalationstechnik, denen - zu Recht - für den Therapieerfolg fast mehr Bedeutung beigemessen wird als der Auswahl der Wirkstoffe per se. Das Device sollte für den Patienten einfach und intuitiv anwendbar sein, um zu gewährleisten, dass die applizierten Wirkstoffe tatsächlich dort ankommen, wo sie wirken sollen. An der Auswahl des Devices sollte der Patient beteiligt werden, damit seine Wünsche und Fähigkeiten optimal berücksichtigt werden. Dass GINA diesen letzten Aspekt, die Patientenwünsche und -skills, so in den Vordergrund rückt, ist neu und sehr zu begrüßen. In der Praxis macht man häufig die Erfahrung, dass eine inhalative Therapie genau daran scheitert, dass der Patient zu wenig in die Entscheidung einbezogen wird. Die Deutsche Atemwegsliga hat auf ihrer Internetseite (www.atemwegsliga.de) Videos für alle in Deutschland erhältlichen Inhala- tionsgeräte veröffentlicht, die in diesem Sinne die Auswahl der Inhalatoren für den Patienten erleichtern können.

Das neue Stufenschema für die Therapie beinhaltet drei wesentliche Änderungen gegenüber der früheren Version:

1. Selbst für leichtes intermittierendes Asthma (Therapiestufe 1) sollte jetzt als mögliche Therapie unter Berücksichtigung des zukünftigen Risikos ein ICS als Dauertherapie in Erwägung gezogen werden. Das ist zwar pathophysiologisch möglicherweise richtig, die Evidenz ist aber nicht eindeutig, dass hierdurch zukünftige Risiken verringert würden [9]. Außerdem heilt ICS selbst bei früher Intervention das Asthma nicht [10]. In der täglichen Praxis werden nur sehr wenige über Monate hinweg beschwerdefreie Patienten bereit sein, eine ICS-Dauertherapie über sich ergehen zu lassen. Es ist auch nicht unumstritten, inwieweit es hierzu bessere Alternativen geben könnte in Form einer intermittierenden ICS-Therapie [11] oder ICS /LABA Kombinationstherapie [12] - Optionen, die gar keine Erwähnung finden.

2. Anders als die intermittierende ICS/LABA-Therapie findet jetzt die mit reichlich klinischer Evidenz belegte MART-Strategie (Maintenance and reliever therapy), also die Verordnung der Kombination von ICS/LABA anstelle eines kurzwirksamen Beta2-Agonisten auch als „Notfallmedikament“, einen prominenten Platz. Auf Therapiestufe 3, 4 und 5 können die Patienten anstelle eines reinen Beta2-Agonisten zusätzlich zur niedrigstmöglichen ICS/LABA-Dauertherapie auch für die Bedarfssituation ein kombiniertes Präparat bekommen mit dem rasch und lang wirksamen Beta2-Agonisten Formoterol und einem niedrig dosierten ICS (Budesonid oder Beclomethason), sodass sie bei akuten Symptomen immer eine ICS-Dosis zusätzlich inhalieren. Dahinter steht bekanntlich die Überlegung, dass Akutsymptome Zeichen einer unzureichenden Entzündungskontrolle darstellen. Von 4 ICS/Formoterol-Kombinationen auf dem deutschen Markt sind 3 für diese Indikation zugelassen. Es ist auf jeden Fall zu begrüßen, dass MART nun auch Eingang in die offiziellen Empfehlungen gefunden hat. Für die Mehrzahl der Asthmapatienten ist das eine relevante Option. 
Tab. 3 Step-down der Controller-Therapie.

Ziel
- die niedrigste Dosis zu finden, die Symptome und Exazerbationen
kontrolliert, und das Nebenwirkungsrisiko zu minimieren
Wann erwägen?
- wenn die Symptome gut kontrolliert sind und die Lungenfunktion
stabil bleibt für $\geq 3$ Monate
- keine Atemwegsinfektionen, keine Reisen und keine Schwangerschaft
geplant
Vorbereitungen
- Symptome dokumentieren und Risikofaktoren abschätzen
- sicherstellen, dass der Patient über einen schriftlichen Asthma-
Aktions-Plan verfügt
- Follow-up-Visite in 1 - 3 Monaten vereinbaren
Step-down mit verfügbaren Präparaten
- ICS-Dosis alle 3 Monate um 25 - $50 \%$ reduzieren, ist bei den meisten
Patienten machbar und sicher
Absetzen des ICS wird bei erwachsenen Asthma-Patienten nicht
empfohlen

3. Auf Therapiestufe 5 wird jetzt für Patienten mit dem Phänotyp IgE-vermitteltes allergisches Asthma die Anti-IgE-Therapie mit Omalizumab als Behandlung der ersten Wahl ausdrücklich noch vor Verordnung von OCS, empfohlen. Hier ist eine kleine Revolution in der personalisierten Medizin für Asthmapatienten eingeleitet worden. Auf Stufe 5 werden in der nahen Zukunft weitere, für umschriebene Phänotypen geeignete personalisierte Therapien verfügbar sein, zum Beispiel für das eosinophile Asthma.

Zuzustimmen ist auch der GINA-Empfehlung, dass auf Stufe 3 die niedrige ICS-Dosis in Kombination mit einem LABA den Vorzug erhält, statt wie beispielsweise die deutsche Nationale Versorgungsleitlinie Asthma (www.versorgungsleitlinien.de/themen/ asthma) die Option eines höher dosierten ICS in Monotherapie als bevorzugte Alternative aufzuführen.

Die Forderung von GINA, bei Erwachsenen das einmal verordnete ICS nicht mehr abzusetzen, ist nicht nur realitätsfremd, sondern meiner Meinung nach schlicht falsch. Es gibt viele Patienten, die in Rahmen von Virusinfekten oder saisonalen Allergieeinflüssen (Pollenasthma) nur für wenige Wochen im Jahr inhalative Kortikosteroide benötigen. Eine kontinuierliche ICS-Zwangsbehandlung solcher Patienten ist weder durchsetzbar noch vernünftig: Schließlich haben auch ICS Nebenwirkungen und verursachen Kosten. Einmal Kortison - immer Kortison würde auch die Akzeptanz seitens der Patienten verschlechtern. Sollte eine als alternative Option auf Therapiestufe 1 begonnene ICS-Therapie wirklich lebenslang fortgesetzt werden?

Für die Praxis hilfreich sind die Hinweise zur Therapieadjustierung, also Step-up und Step-down, in denen auch die Abstände beschrieben sind, in denen die Qualität der Asthmakontrolle überprüft werden sollte: Bei bereits therapierten stabilen Patienten alle 3-12 Monate, bei Schwangeren alle 4-6 Wochen, nach Exazerbation binnen 1 Woche. Vor allem die ausführlichen Erläuterungen zur Step-down-Strategie ( Tab.3) werden viele Kolle- gen als sehr praxisrelevant und hilfreich empfinden, da bislang solche Empfehlungen fehlten.

Als Fazit bleibt, dass angesichts der diagnostischen Unsicherheiten - Stichwort: Abgrenzung gegen COPD und ACOS -, aber auch der Unterdiagnose und Überdiagnose des Asthmas sowie der zunehmenden Zahl phänotypisch ausgerichteter Therapien Asthmapatienten künftig noch stärker der fachärztlichen Kompetenz bedürfen, um individuell optimal und leitliniengerecht behandelt zu werden.

\section{Danksagung \\ $\nabla$}

Der Autor dankt Frau Manuela Arand, Berlin, für die redaktionelle Bearbeitung.

\section{Interessenkonflikt}

$\nabla$

P. Kardos hat wirtschaftliche Verbindungen mit folgenden Firmen: AstraZeneca, Bionorica, Boehringer Ingelheim, Chiesi, GlaxoSmithKline, Mundipharma, Novartis, Schwabe, Takeda und Menarini.

\section{Literatur}

1 Chung KF, Wenzel SE, Brozek JL et al. International ERS/ATS guidelines on definition, evaluation and treatment of severe asthma. Eur Respir J 2014; 43: 343-373

2 Magadle R, Berar-Yanay N, Weiner $P$ et al. The risk of hospitalization and near-fatal and fatal asthma in relation to the perception of dyspnea. Chest 2002; 121: 329-333

3 Silva CM, Barros L. Asthma knowledge, subjective assessment of severity and symptom perception in parents of children with asthma. J Asthma 2013; 50: 1002-1009

4 Aaron SD, Vandemheen KL, Boulet $L P$ et al. Overdiagnosis of asthma in obese and nonobese adults. CMAJ 2008; 179: 1121 -1131

5 Lucas AE, Smeenk FW, Smeele IJ et al. Overtreatment with inhaled corticosteroids and diagnostic problems in primary care patients, an exploratory study. Fam Pract 2008; 25: 86-91

6 Montnemery P, Hansson L, Lanke J et al. Accuracy of a first diagnosis of asthma in primary health care. Fam Pract 2002; 19: 365-368

7 Marklund B, Tunsater A, Bengtsson C. How often is the diagnosis bronchial asthma correct? Fam Pract 1999; 16: 112-116

8 Travers J, Marsh S, Williams $M$ et al. External validity of randomised controlled trials in asthma: to whom do the results of the trials apply? Thorax 2007; 62: 219-223

9 Guilbert TW, Morgan WJ, Zeiger RS et al. Long-Term Inhaled Corticosteroids in Preschool Children at High Risk for Asthma. N Engl J Med 2006; 354: 1985-1997

10 Haahtela T, Jarvinen M, Kava $T$ et al. Effects of reducing or discontinuing inhaled budesonide in patients with mild asthma. N Engl J Med 1994; 331: 700-705

11 Boushey HA, Sorkness CA, King TS et al. Daily versus As-Needed Corticosteroids for Mild Persistent Asthma. N Engl J Med 2005; 352: $1519-1528$

12 Papi A, Canonica GW, Maestrelli P et al. Rescue Use of Beclomethasone and Albuterol in a Single Inhaler for Mild Asthma. N Engl J Med 2007; 356: $2040-2052$ 